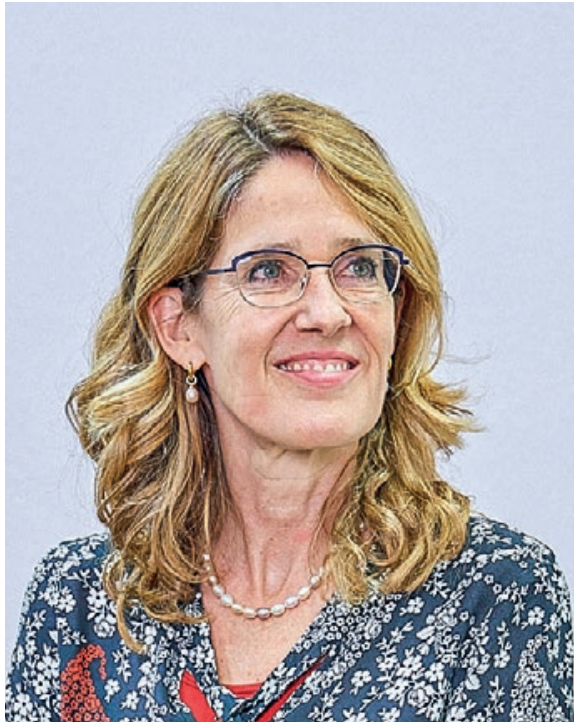

Dr. med. Rachel Enz Perschel

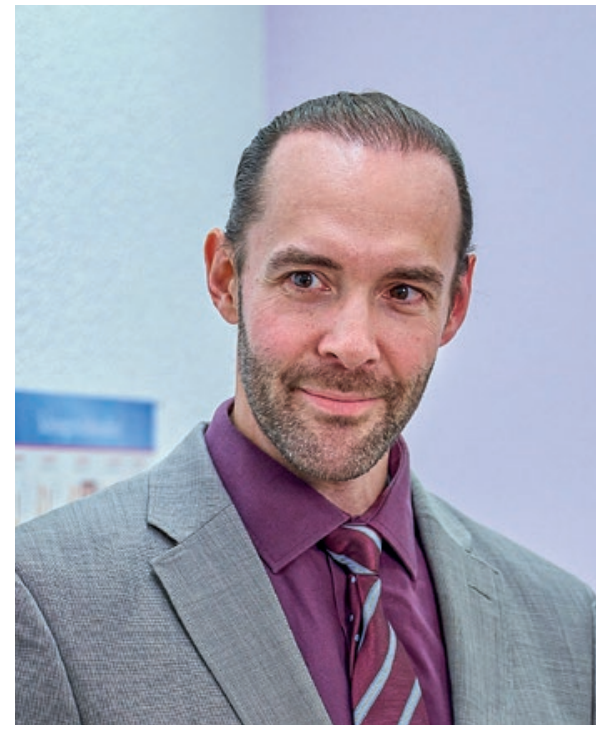

Dr. iur. Kaspar Gerber

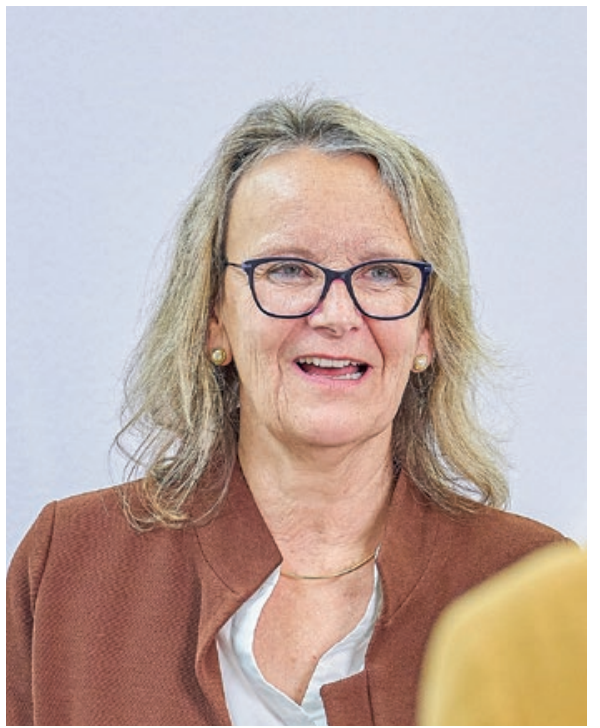

Dr. theol. Ruth Baumann-Hölzle

\title{
Impfen im Spannungsfeld von Druck und Gegendruck
}

Interview: Charlotte Schweizer

Leiterin Kommunikation FMH

Die Unzufriedenheit mit den Coronamassnahmen steigt. Hohe Impfquoten versprechen die Aufhebung der Einschränkungen, vermehrt wird über ein Impfobligatorium für einzelne Institutionen oder Berufsgruppen gesprochen. Dies wirft viele Fragen auf. Im folgenden Interview legen eine Ärztin, eine Medizinethikerin und ein Rechtswissenschaftler ihre persönlichen Standpunkte dar. Die FMH will damit der Komplexität des Themas Rechnung tragen und zu Reflexion und differenziertem Dialog einladen.

Gibt es in einer Pandemie so etwas wie eine moralische Verpflichtung, dass man sich impfen lässt, Frau Baumann-Hölzle? Kann man Menschen zu einer Impfung zwingen?

Baumann-Hölzle: In der Rechtswissenschaft erfüllt jede Form von medizinischer Handlung den Tatbestand einer Körperverletzung. Ihr Pendant in der Ethik ist die Integritätsverletzung. Es sind Situationen denkbar, wo sich eine Impfpflicht von der Verhältnismässigkeit her für bestimmte Gruppen oder gar für die Bevölkerung aufdrängt. Bis jedoch der demokratisch verbriefte Staat eine Impfpflicht verordnen kann, muss sehr sorgfältig abgewogen werden zwischen dem Anspruch des Einzelnen auf physische, psychische und

\section{Am Interview nahmen teil:}

Dr. med. Rachel Enz Perschel, Fachärztin für Allgemeine Innere Medizin, Ärztliche Leiterin wollimed ag Arztpraxis Dr. theol. Ruth Baumann-Hölzle, Expertin für Ethik im Gesundheitswesen, Leiterin Stiftung Dialog Ethik Dr. iur. Kaspar Gerber, LL.M, Wissenschaftlicher Mitarbeiter (postdoc), Lehrstuhl für Staats-, Verwaltungs- und Sozialversicherungsrecht, Universität Zürich

Das Interview fand am 10. November 2021 statt.

soziale Integrität gegenüber einem Impfzwang und dem Anspruch auf Schutz vor Fremgefährdung anderer Menschen. Auch dieser Schutzanspruch wird letztlich mit diesem vielfältigen Integritätsanspruch be- 
gründet. In einer solchen Situation befinden sich die Staatsakteure in einem ethischen Dilemma. Wie auch immer sie entscheiden, es kommt stets zu Grundrechtsverletzungen. Prinzipiell stellt sich bei der Integritätsverletzung die Frage, wie stark sie ist und ob es niederschwelligere Alternativen dazu gibt. So hat die Testpflicht eine deutlich geringere Eingriffstiefe als die Impfung und muss daher zuerst ausgeschöpft werden. Erst wenn das Testen Übertragungen nicht nachweislich in vergleichbarer Art und Weise wie ein Impfung verhindert, kann eine Impfpflicht ethisch vertretbar sein.

\section{Wie sieht es aus rechtlicher Sicht aus? Darf der Staat Menschen zum Impfen zwingen, Herr Gerber?}

Gerber: Ein Impfobligatorium ist unter bestimmten Bedingungen rechtlich zulässig und vertretbar, so steht es im Epidemiengesetz. Es nennt als Voraussetzung die Verhältnismässigkeit und die Subsidiarität. Eine Massnahme ist dann verhältnismässig, wenn sie geeignet, notwendig und zumutbar ist. In der jetzigen besonderen Lage kann der Bundesrat ein Impfobligatorium anordnen für gefährdete Bevölkerungsgruppen, für besonders exponierte Personen und für Personen, die bestimmte Tätigkeiten ausüben. Die Verordnung führt aus, dass eine Impfpflicht nur sehr gezielt eingesetzt werden soll, also beispielsweise beim Gesundheitspersonal nur in denjenigen Bereichen, in welchen z.B. das Risiko einer Weiterverbreitung der Krankheit erhöht ist. Wobei auch hier die Subsidiarität gilt, also testen statt impfen. Denn natürlich kann man niemanden physisch zur Impfung zwingen.

\section{Dr. med. Rachel Enz Perschel: "Wer sich eine Meinung gebildet hat, ist mit medizinischen Argumenten kaum mehr zu erreichen.»}

\section{Wie ist die Covid-Impfung von Ihren Patientinnen und} Patienten aufgenommen worden, Frau Enz Perschel?

Enz Perschel: In meiner Wahrnehmung sind etwa 80 Prozent unserer Patientinnen und Patienten über 50 geimpft. Die totale Ablehung der Impfung erlebe ich selten. Anfangs wurden wir von den Impfwilligen geradezu überrannt, und aktuell wird z.T. vehement nach dem Booster verlangt. Einige Schwangere sehnen den vierten Monat herbei, damit sie sich impfen lassen können. Bei den über 80-jährigen Menschen gibt es die gesunde und vitale Gruppe, die sich unbedingt impfen lassen will. Daneben gibt es jene Betagten, die müde sind und sagen, dass sie lange genug gelebt haben und nun eine Ansteckung in Kauf nehmen. Einige der über 90-Jährigen lassen sich sogar ganz bewusst nicht impfen. Andere Impfunwillige be- trachten sich schlicht als nicht gefährdet. Sie begründen dies damit, dass sie daheim seien und wenig Leute treffen würden; oder sie denken, dass sie gute Abwehrkräfte haben.

Dr. iur. Kaspar Gerber: «Ein Impfobligatorium kann Abwehrreaktionen, Umgehungsverhalten oder Personalverluste provozieren.»

Aus epidemiologischer Sicht sollen strikte Massnahmen die Pandemie möglichst rasch eindämmen, aus individueller Sicht sollen die persönliche Freiheit und Selbstbestimmung möglichst nicht tangiert werden. Wie schätzen Sie diesen Zielkonflikt ein?

Gerber: Ganz allgemein sind Nutzen und Schaden von einzelnen Pandemiemassnahmen laufend $\mathrm{zu}$ analysieren und gegeneinander abzuwägen. Ein Impfobligatorium bzw. auch das Zertifikat erzeugen Druck, denn Nichtgeimpften entstehen dadurch Nachteile: So wird ihnen beispielsweise der Zugang zu bestimmten öffentlichen Orten wie Restaurants, Fitnesscenter oder Altenheimen verwehrt. Möglicherweise ist bei einer arbeitsvertraglichen Impfpflicht auch ihre Arbeitstätigkeit gefährdet, falls der Arbeitgeber das Testen nicht zulässt. Neben dem epidemiologischen Aspekt gilt es zu berücksichtigen, dass ein Impfobligatorium Abwehrreaktionen provozieren kann, Umgehungsverhalten oder auch Personalverluste. Meines Erachtens werden gewisse Massnahmen wie die Zugänglichkeit zu öffentlichen Institutionen momentan zu pauschal eingesetzt statt spezifischer direkt auf die Risikogruppen und die epidemiologisch relevanten Orte.

Baumann-Hölzle: In der Ethik unterscheiden wir die Aktivitäten des alltäglichen und des guten Lebens. Eingriffe ins Alltagsleben, wie nicht mehr einkaufen oder zur Schule gehen dürfen, erachten wir als massive soziale Integritätsverletzung; hingegen werden Eingriffe ins gute Leben, etwa durch das Verbot eines Restaurantbesuchs, als weniger gravierend eingestuft. Gleichwohl stellt sich die Frage, wie weit man dabei den gesellschaftlichen Frieden nicht aufs Spiel setzt, denn es geht nicht nur um Gesundheit, sondern wie erwähnt um viele weitere Werte wie z.B. gesellschaftlichen $\mathrm{Zu}$ sammenhalt oder Anspruch auf Bewegungsfreiheit. Deshalb ist es sehr wichtig, nicht von einer allgemeinen Impfpflicht zu sprechen, sondern das Risiko einer Ansteckung in Relation zu anderen Risiken zu sehen und die Massnahmen entsprechend den unterschiedlichen Risikogruppen zu differenzieren. Diese Auseinandersetzung vermisse ich gegenwärtig im öffentlichen Diskurs. Enz Perschel: Je mehr Menschen geimpft sind, desto schneller ist die Pandemie beendet. Das Testen ver- 


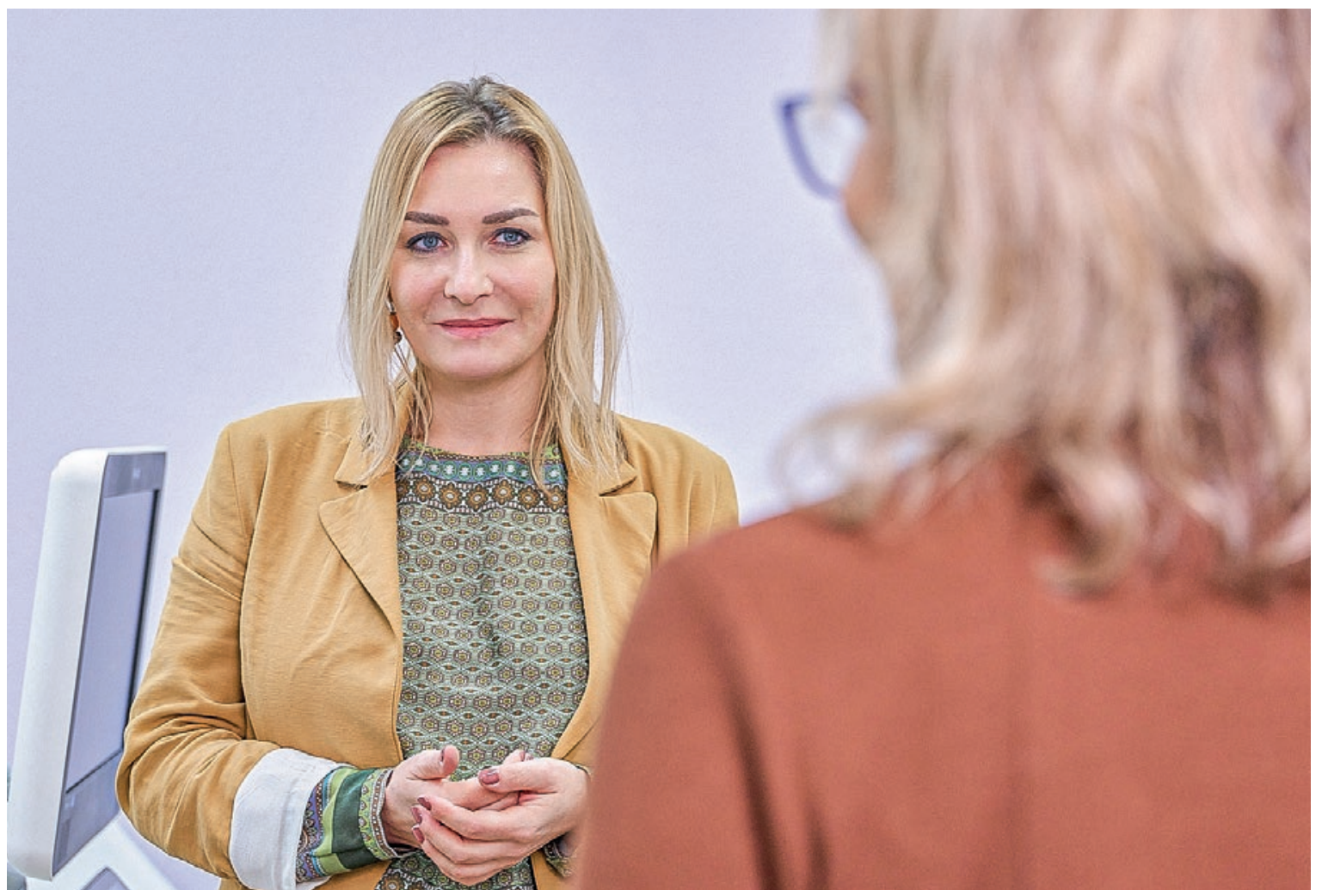

Charlotte Schweizer im Gespräch mit Dr. theol. Ruth Baumann-Hölzle.

kürzt sie nicht. Am liebsten hätten die Leute keine Impfung und keine Maskenpflicht. Es gibt im Moment jedoch keine Alternative dazu. Information und Empfehlungen sind der einzige Weg, unsere Mitmenschen von der Impfung zu überzeugen. Mein Eindruck ist jedoch, dass man diejenigen, die sich ihre Meinung gebildet haben, mit medizinischen Argumenten kaum mehr erreicht.

\section{Kleine Kinder haben ein geringes Risiko, aufgrund einer Covid-Infektion bleibende Schäden davonzutra- gen. Wenn man sie impft, geschieht dies primär, um andere gefährdete Menschen vor einer Ansteckung zu schützen. Was sagen Sie zu dieser Abwägung?}

Enz Perschel: Wir wissen heute noch nicht, wie sich der Impfstoff auf die Kinder auswirkt. Ich rate den Eltern, sich zu impfen, so dass sie mehrheitlich vor Ansteckung durch ihre Kinder geschützt wären und selbst nicht in Quarantäne müssen. So brauchten die Kinder auch keine Angst davor zu haben, ihre Eltern oder Grosseltern anzustecken. Die Forderung der Elternorganisation «Protect the Kids» nach der Impfung für unter 12-Jährige kann ich teilweise nachvollziehen, vor allem wenn es um die Hochrisikokinder mit Herzoder Lungenerkrankungen geht, die ja auch gegen Grippe geimpft werden. Bei ihnen kann eine Covid-
Impfung (zum aktuellen Stand vom November 2021) im Off-Label-Use erwogen werden. In anderen Ländern ist der Impfstoff bereits für 5- bis 11-Jährige zugelassen. Gerber: Momentan sehe ich den medizinischen Nutzen von generellen Covid-19-Impfungen bei Kindern nicht, ausser bei gewissen Vorerkrankungen. Ich finde es heikel, ihnen pauschal zu sagen: Ihr seid kleine "Virenschleudern", deshalb impfen wir euch - auch wenn ihr selbst nur ein minimes Risiko habt, ernsthaft zu erkranken.

Baumann-Hölzle: Es wäre eine Instrumentalisierung der Kinder für die ungeimpften Eltern oder Impfunwilligen aus den Risikogruppen. Das kann es nicht sein: Die Risikogruppen hatten die Möglichkeit, sich impfen zu lassen. Ein weiterer problematischer Punkt: Wie kann ein Kind, das noch keinen Lebensentwurf entwickeln konnte, in Bezug auf eine Impfung wirklich eine informierte Entscheidung treffen, allenfalls gegen den Willen der Eltern? Diese Frage kann einen Keil zwischen Eltern und Kind treiben.

\section{Was sind Ihrer Meinung nach die Gründe für Impfskepsis?}

Enz Perschel: Vor allem zu Beginn der Pandemie war es ungünstig, dass man sich durch die Behörden oft schlecht oder gar falsch informiert sah. Ich erinnere 
an die bald revidierte Botschaft des BAG «Masken nützen nichts», gefolgt von mangelnder Versorgung mit Masken und weiterem Schutzmaterial. So haben sich Gruppierungen gebildet, die nicht mehr alles glauben. Weitere Gründe sind Angst und mangelndes Wissen. So fürchten sich viele junge Menschen vor einer Herzmuskelentzündung, die durch die Impfung ausgelöst werden kann - ohne zu sehen, dass eine Coronavirus-Infektion häufiger als die Impfung für eine Herzmuskelentzündung verantwortlich sein kann. Oder das Märchen der Unfruchtbarkeit, welches auch das wissenschaftlich fundierte Videostatement auf der BAG-Homepage offensichtlich nicht entkräften kann.

Baumann-Hölzle: Sie bringen mich auf Periodenverschiebungen, die viele Frauen nach der Impfung erlebten und die von ärztlicher bzw. auch behördlicher Seite oft nicht ernst genommen wurden. Daraus könnte man Folgendes ableiten: Wenn solche Vorkommnisse nicht ernst genommen werden, vielleicht ist dann an der Geschichte mit der Unfruchtbarkeit doch etwas dran? Ich will damit sagen, dass etliche kommunikative und inhaltliche Fehler passiert sind, die das Vertrauen vieler Menschen in die Behörden nachhaltig gestört haben. Der Bundesrat hat beispielsweise mehrmals gesagt: Wenn alle Impfwilligen und Risikopatienten geimpft sind, dann werden wir die Massnahmen aufheben. Und jetzt, wo dies der Fall ist, heisst es: Wir müssen die Massnahmen trotzdem beibehalten, weil sonst die Intensivstationen überlastet werden.

\section{Dr. theol. Ruth Baumann-Hölzle: «Ob wir es schaffen, die medizinethischen Grundsätze nach der Pandemie wieder zu aktivieren?»}

Gerber: Selbstverständlich ist die Impfung für bestimmte Personengruppen gegenwärtig eine wichtige und innert weniger Wochen sehr wirksame Präventionsmassnahme. Allerdings wurde und wird die Impfung als "Gamechanger" der Pandemie proklamiert, obwohl schnell bekannt wurde, dass sie als dies auch international betrachtet nicht so absolut gelten kann. Es ist deshalb nachvollziehbar, dass einige Leute - geimpfte und ungeimpfte - frustriert sind. Ein grundsätzliches Problem scheint mir, dass die Botschaft der Impfkampagne eher lautet «du musst die anderen schützen» statt «schütze dich selbst». Dies erzeugt Druck, sich im Namen der Solidarität impfen zu lassen, was zu Aversionen und Gegendruck führt. Das hat meines Erachtens nichts mit gesellschaftsschädlichem Egoismus zu tun; der Selbstschutz entspricht schlicht dem Hauptzweck einer Impfung.
Frank Ulrich Montgomery, der Vorstandsvorsitzende des Welt-Ärztebundes, sprach von einer "Tyrannei der Ungeimpften über die zwei Drittel Geimpften", die allen anderen Einschränkungen aufzwingen. Wie beurteilen Sie diese Aussage?

Enz Perschel: Geimpfte sind gemäss aktuellem Wissen weniger infektiös, wenn sie angesteckt sind. Die Impfung ist kein hundertprozentiger Schutz, insofern können auch Geimpfte der Allgemeinheit das Maskentragen und Abstandhalten nicht ersparen.

Dr. med. Rachel Enz Perschel: «Geimpfte sind gemäss aktuellem Wissen weniger infektiös, wenn sie angesteckt sind.»

Gerber: Vor dem Hintergund, dass die Fallzahlen trotz Impfung steigen und andere Länder mit z.T. sehr hoher Impfquote die Massnahmen nach und nach wieder verschärfen, ist Herrn Montgomerys Aussage problematisch. Er kollektiviert die Verantwortung für Gesundheit, also den Fremdschutz. So wird die Impfung quasi $\mathrm{zu}$ einem politischen und gesellschaftlichen Statement. Geimpftsein wird zu einem Gütesiegel, welches die Leute teilweise auch in den sozialen Medien stolz mittels zweier Spritzen präsentieren. So spitzt sich die Polarisierung zu, bis man sagt: Du bist geimpft oder nicht geimpft, deswegen bist du für oder gegen das Covid-Gesetz oder für oder gegen den Bundesrat. Der Impfstatus wird wahnsinnig heraufstilisiert.

Baumann-Hölzle: Herr Montgomery müsste sich mit seiner Kritik mit weniger aggressiven Worten auf Gruppen fokussieren, die sich trotz hohen Risikos nicht impfen lassen, statt flächendeckend alle Ungeimpften als tyrannisch zu bezeichnen. So kommt es zur Verurteilung von Menschen, ohne deren Beweggründe zu kennen. Hier hat insofern bereits eine Eskalation stattgefunden, als dass Andersdenkende schon als Gegner, ja Feinde behandelt werden. Solch pauschalisierende Aussagen sind sehr gefährlich, weil sie unterschiedlich denkende Bevölkerungsgruppen gegeneinander aufwiegeln.

\section{Es gibt öffentliche Institutionen, in denen der Impf} bzw. Teststatus mit goldenen bzw. silbernen Ansteckern gekennzeichnet wird. Wie denken Sie darüber? Gerber: Sowohl Impf- wie Teststatus sind eigentlich immer noch Privatsache. Dieser Schutz der Privatsphäre ist bereits durch das Zertifikat massiv aufgeweicht.

Baumann-Hölzle: Hinter den goldigen und silbernen Ansteckern steht die These: Mit der Impfung haben wir alles im Griff, was nicht zutrifft. So besteht keinerlei Berechtigung, die Segregation von Geimpften und Ungeimpften in dieser Art und Weise voranzutreiben. Ich stelle fest, dass in der Pandemie viele Grundsätze 
der Medizinethik ausgehebelt werden, d.h., sie werden nicht mehr in dem Masse respektiert, wie sie sollten. Es ist zum Beispiel höchst bedenklich, wie das Arztgeheimnis aufgelöst und Arbeitgebende Gesundheitsdaten der Mitarbeitenden erfragen dürfen. Und ich frage mich, ob wir es schaffen, die medizinethischen Grundsätze nach der Pandemie wieder zu aktivieren.

\section{Was sind für Sie die grössten Herausforderungen für} unsere Gesellschaft bei der Eindämmung der Pandemie?

Gerber: Bei respiratorischen Viren handelt es sich grundsätzlich um ein alltägliches und saisonal akzentuiertes Lebensrisiko. Allgemein gültige Gegenmassnahmen wie das "social distancing» können daher je nach deren Intensität sofort gesamtgesellschaftliche Kollateralschäden bewirken. Im Moment bindet das staatliche Risikomanagment der Pandemie sehr viele Mittel; irgendwann ist der Punkt erreicht, wo deshalb andere Lebensrisiken und die von ihnen Betroffenen zu lange zu kurz gekommen sind; dort wird es schwierig mit der Verallgemeinerungsfähigkeit von Massnahmen und folglich mit der Rechtsgleichheit. Wir sollten nach und nach dazu übergehen, über das Lebensrisiko Coronavirus primär zu informieren und allenfalls auch künftig differenzierte Impfempfehlungen auszusprechen.

Enz Perschel: Die Jungen leiden besonders unter den Massnahmen und werden auch an den Folgen der Pandemie noch lange zu tragen haben. Aktuell sehen wir eine Zunahme von psychischen Problemen vor allem bei den Jugendlichen.

Baumann-Hölzle: Die Generationengerechtigkeit ist eines von vielen Gerechtigkeitsproblemen, die wir schon lange hatten und nun durch die Pandemie an die Oberfläche gespült werden. Und sicher bietet eine Gesellschaft, die Gerechtigkeitsprobleme in diesem Ausmass zulässt - die Ungleichbehandlung von Krankheitsbetroffenen, belastende Arbeitsbedingungen, gerade im Gesundheitswesen -, den idealen Nährboden für Aggression. Moralische Verfehlungen führen an gewissen Orten bereits zu moralischem Stress, moralischen Verletzungen und gar Zusammenbrüchen - man kann sich nicht mehr in den anderen hineinversetzen, hört sich nicht mehr zu, verschliesst sich faktenbasierten Argumenten. Daran müssen wir als Gesellschaft dringend arbeiten. Und ganz sicher

\section{Dr. med. Rachel Enz Perschel: "Aktuell sehen wir eine Zunahme von psychischen Problemen vor allem bei Jugendlichen.»}

muss der Staat versuchen, das Vertrauen jener Menschen zurückzugewinnen, das er u.a. durch nicht eingelöste Versprechen und mangelnde Transparenz erschüttert hat.

Dies ist der dritte und letzte Teil der Kurzreihe zum Thema Impfobligatorium. In Teil 1 schrieb Dr. iur. Iris HerzogZwitter über das Thema «Impfobligatorium - Der rechtliche Rahmen» (Schweiz Ärzteztg. 2021;102(47):1556-9). Dr. iur. Kaspar Gerber erörterte in Teil 2 das Thema «Covid-19: Verhältnismässigkeit von Massnahmen zur Impfmotivation» (Schweiz Ärzteztg. 2021;102(48):1599-1601).

Bildnachweis Christian Jäggi 